\title{
ZOOSYMPOSIA
}

https://doi.org/10.11646/zoosymposia.18.1.1

http://zoobank.org/urn:lsid:zoobank.org:pub:5BF04345-F17F-4CDD-BE10-4C193BF2714F

18

\section{Proceedings of the 16th International Symposium on Trichoptera}

PONGSAK LAUDEE \& JOHN C. MORSE (Eds)

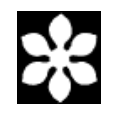

Magnolia Press

Auckland, New Zealand 
PONGSAK LAUDEE \& JOHN C. MORSE (Eds)

Proceedings of the 16th International Symposium on Trichoptera

(Zoosymposia 18)

$201 \mathrm{pp}$.

12 June 2020

ISBN 978-1-77670-951-9 (paperback)

ISBN 978-1-77670-952-6 (online edition)

FIRST PUBLISHED IN 2020 BY

Magnolia Press

P.O. Box 41-383

Auckland 1346

New Zealand

e-mail: zoosymposia@mapress.com

https://www.mapress.com/j/zs

Series editor, ZHI-QIANG ZHANG

ISSN 1178-9905 (Print edition)

ISSN 1178-9913 (Online edition) 